\title{
Glutamatergic Afferents from the Hippocampus to the Nucleus Accumbens Regulate Activity of Ventral Tegmental Area Dopamine Neurons
}

\author{
Stan B. Floresco, Christopher L. Todd, and Anthony A. Grace \\ Departments of Neuroscience and Psychiatry, University of Pittsburgh, Pittsburgh, Pennsylvania 15260
}

Several studies have shown that the mesolimbic dopamine (DA) system is strongly influenced by the ventral subiculum (vSub) of the hippocampus. To examine whether this occurs by activation of DA neuron firing, the effects of chemical stimulation of the vSub on ventral tegmental area (VTA) DA neuron activity were examined using extracellular single-unit recordings. Infusions of NMDA into the vSub increased the number of spontaneously firing DA cells recorded per electrode track, while having no effect on firing rate or burst firing. This response was abolished by intranucleus accumbens (NAc) infusions of the glutamate receptor antagonist kynurenic acid. This effect did not involve the prefrontal cortex, because infusions of tetrodotoxin into the prefrontal cortex did not affect the increase in spontaneously active DA cells. Infusions of either kynurenic acid into the NAc or tetrodotoxin into the vSub decreased the firing rate and burst firing of DA neurons without altering the number of spontaneously active DA neurons. These data show that glutamatergic afferents from the vSub to the NAc exert a potent excitatory effect on VTA DA neurons, influencing both DA neuron population activity and the regulation of the firing properties of these neurons. As a result, dysfunctions in hippocampal circuitries may contribute to the hyperexcitable state of the DA system that is present in schizophrenia.

Key words: ventral subiculum; ventral tegmental area; nucleus accumbens; NMDA; glutamate; dopamine; rat
The nucleus accumbens (NAc) is a central component of the basal ganglia that is positioned to integrate signals arising from limbic and cortical areas and to modulate motor output related to goal-directed behavior (Groenewegen et al., 1991; Mogenson et al., 1993). A prominent, excitatory glutamatergic input to the medium spiny neurons of the NAc arises from the ventral CA1/ subiculum (vSub) region of the hippocampus (Groenewegen et al., 1987; Brog et al., 1993), and these inputs are in close apposition to dopaminergic projections arising from the ventral tegmental area (VTA) (Totterdell and Smith, 1989; Sesack and Pickel, 1990). Electrophysiological studies have demonstrated that mesoaccumbens dopamine (DA) transmission exerts a powerful neuromodulatory influence over hippocampal inputs to the NAc. Stimulation of the VTA, or direct activation of DA receptors by exogenous application of DA agents, can either inhibit or facilitate neural activity of NAc neurons driven by hippocampal input (Yang and Mogenson, 1984, 1986, 1987; DeFrance et al., 1985; Pennartz et al., 1992, 1994; Gonon and Sundstrom, 1996). Moreover, behavioral studies have shown that DA transmission in the NAc also exerts modulatory control over behaviors that are mediated by hippocampal-ventral striatal circuits (Burns et al., 1993, 1996; Wu and Brudzynski, 1995; Floresco et al., 1996; Bardgett and Henry, 1999; Floresco and Phillips, 1999).

Recent neurochemical studies show that the hippocampus in

Received Jan. 23, 2001; revised April 17, 2001; accepted April 17, 2001.

This work was supported by United States Public Health Service Grants MH 01055, MH 57440, and MH 45156. S.B.F. is a recipient of a Human Frontiers Science Organization postdoctoral fellowship. We thank Drs. Holly Moore and Anthony West for their assistance and Nicole MacMurdo for her assistance with histology.

Correspondence should be addressed to Dr. Stan B. Floresco, Department of Neuroscience, University of Pittsburgh, 446 Crawford Hall, Pittsburgh, PA 15260. E-mail: floresco@brain.bns.pitt.edu.

Copyright () 2001 Society for Neuroscience $0270-6474 / 01 / 214915-08 \$ 15.00 / 0$ turn can influence DA release in the ventral striatum. Thus, chemical or electrical stimulation of the vSub produces robust and sustained increases in extracellular DA levels in the NAc (Blaha et al., 1997; Brudzynski and Gibson, 1997; Legault and Wise, 1999; Taepavarapruk et al., 2000). However, the mechanisms by which these effects occur remain in dispute. The ability to abolish vSub-stimulated mesoaccumbens DA efflux by blockade of glutamate receptors in the NAc suggests that these increases in DA release may be mediated primarily by glutamate receptormediated mechanisms localized within the ventral striatum (Blaha et al., 1997; Taepavarapruk et al., 2000). In contrast, Legault et al. (2000) have shown that the increase in mesoaccumbens DA after infusion of NMDA into the vSub can be blocked by application of glutamate antagonists in the VTA. The observation that this increase is accompanied by an increase in DA efflux in the VTA and changes in DA neuron firing suggests that the vSub may also modulate NAc DA via changes in DA neuron activity.

One caveat in this analysis is the lack of evidence of a direct projection from the hippocampus to the VTA. As such, it is likely that the modulation of DA cell body activity by the vSub occurs via a polysynaptic circuit. As noted above, the vSub sends a dense projection to the NAc (Groenewegen et al., 1987; Brog et al., 1993). Moreover, the NAc can influence DA neuron activity by both a direct projection to the VTA and an indirect projection via the ventral pallidum (Zahm and Heimer, 1990). The vSub also sends a projection to the medial prefrontal cortex (mPFC) (Conde et al., 1995), which in turn sends excitatory glutamatergic projections to the VTA, although this projection only innervates the mesocortical DA neurons (Carr and Sesack, 2000). Because of these anatomical considerations, the present study was conducted to assess the role of the vSub in the modulation of firing activity of DA neurons in the VTA and the neural circuits that mediate 
these effects. Initial extracellular recording experiments used inf usions of either NMDA or tetrodotoxin (TTX) into the vSub to assess the effects of stimulation or inactivation of the vSub on the spontaneous activity and firing characteristics of DA neurons. Subsequent pharmacological manipulations of the NAc and the mPFC were conducted to elucidate the potential neural pathways by which the vSub would influence mesolimbic DA neuron activity. Understanding the mechanisms by which the hippocampus may modulate DA cell body activity may have important implications for understanding the etiology of schizophrenia, which has been proposed to be linked to both hippocampal dysfunction and hyperexcitability of the DA system (Bogerts, 1993).

\section{MATERIALS AND METHODS}

Subjects and surgery. All experiments were performed on male Sprague Dawley rats (Hilltop, Scottsdale, PA). Animal care and surgical procedures were performed in accordance with the guidelines outlined in the National Institutes of Health Guide for the Care and Use of Laboratory Animals and were approved by the Institutional Animal Care and Use Committee of the University of Pittsburgh. Rats weighing between 290 and $450 \mathrm{gm}$ were deeply anesthetized with chloral hydrate $(400 \mathrm{mg} / \mathrm{kg}$, i.p.) and placed in a stereotaxic apparatus (Narishige, Tokyo, Japan), with the incisor bar set at $-3.3 \mathrm{~mm}$. Core body temperature was monitored by a rectal probe and maintained at $35^{\circ} \mathrm{C}$ by a thermostatically controlled heating pad. Supplemental anesthetic was administered intraperitoneally by constant infusion via a syringe pump (Razel model A-99) at a rate of $10-30 \mathrm{mg} \cdot \mathrm{kg}^{-1} \cdot \mathrm{hr}^{-1}$. In all surgical preparations, the scalp was incised, and holes were drilled in the skull overlying the VTA and the vSub region of the hippocampus. All rats were implanted with 23 gauge guide cannulas directed to a location $\sim 2 \mathrm{~mm}$ dorsal to the vSub (anteroposterior, $-6.0 \mathrm{~mm}$ from bregma; mediolateral, $+5.3 \mathrm{~mm}$ from the midline; and dorsoventral, $4.0 \mathrm{~mm}$ from the dura). Subsets of rats were also implanted with guide cannulas directed $\sim 1 \mathrm{~mm}$ dorsal to either the NAc (anteroposterior, $+1.4 \mathrm{~mm}$ from bregma; mediolateral, $1.3 \mathrm{~mm}$ from the midline; and dorsoventral, $-6.0 \mathrm{~mm}$ from the dura) or the mPFC (anteroposterior, $+3.0 \mathrm{~mm}$ from bregma; mediolateral, $+0.7 \mathrm{~mm}$ from the midline; and dorsoventral, $-3.0 \mathrm{~mm}$ from the dura).

Drugs and drug microinfusions. NMDA $(0.25$ and $0.75 \mu \mathrm{g})$, TTX (1 $\mu \mathrm{M})$, the broad-spectrum glutamate receptor antagonist kynurenic acid $(10 \mu \mathrm{g})$, and Dulbecco's buffer were all obtained from Sigma (St. Louis, MO). NMDA was dissolved in Dulbecco's buffer, TTX was dissolved in deionized water, and kynurenic acid was dissolved in one drop of $0.1 \mathrm{M}$ $\mathrm{NaOH}$, to which Dulbecco's buffer was added. The $\mathrm{pH}$ of the kynurenic acid solution was then adjusted to $\sim 7.4$ with $0.1 \mathrm{M} \mathrm{HCl}$. The doses of NMDA used in the present study were similar to those used by Legault and Wise (1999), and the dose of kynurenic acid was the same as that used by Blaha et al. (1997).

NMDA or TTX was infused into the vSub through a 30 gauge injection cannula that protruded $2.0 \mathrm{~mm}$ past the end of the guide. Infusions were delivered at an injection volume of $0.5 \mu$ l over 2 min. Subsets of rats received intra-NAc infusions of kynurenic acid at a rate of $1.0 \mu \mathrm{l}$ over $1 \mathrm{~min}$ or intra-mPFC infusions of TTX at a rate of $0.5 \mu \mathrm{l}$ over $1 \mathrm{~min}$ via an injection cannula that protruded $0.8 \mathrm{~mm}$ past the end of the guide. In these experiments, infusions into the NAc or the mPFC were administered 3 min before infusions of NMDA into the vSub.

Extracellular recordings and experimental protocol. Extracellular recording microelectrodes were constructed from 2.0-mm-outer-diameter borosilicate glass capillary tubing (WPI) using a vertical micropipette puller (Narishige). The tips of the electrodes were broken back against a glass rod to an $\sim 1 \mu \mathrm{m}$ tip diameter and filled with $2 \mathrm{M} \mathrm{NaCl}$ containing $2 \%$ Pontamine sky blue dye. The in vitro impedance of the microelectrodes ranged from 5 to $10 \mathrm{M} \Omega$ as measured at $135 \mathrm{~Hz}$ using a Winston Electronics BL-1000 impedance meter. After drilling a burr hole overlying the VTA, the dura was resected, and the electrode was lowered into the VTA (coordinates, $+3.6 \mathrm{~mm}$ anterior from lambda, $1.0 \mathrm{~mm}$ lateral from the midline, and $6.5-9 \mathrm{~mm}$ ventral from the brain surface) with a hydraulic microdrive (Kopf model 640). The electrode signal was amplified, filtered, and discriminated from noise using a combination amplification and window discrimination unit for extracellular recording (Fintronics, Orange, CT) and displayed on an oscilloscope (Tektronics, Wilsonville, OR). The data were acquired, stored, and analyzed using custom-designed computer software (Neuroscope) running on an Intel- based personal computer with a data acquisition board interface (Microstar Laboratories, Bellevue, WA).

Immediately after infusions into the vSub, a recording electrode was lowered into the VTA. The electrophysiological properties of spontaneously active DA neurons were sampled in the VTA by making six to nine vertical passes of the electrode through the DA cell body region. These tracks were made in a predefined pattern, with each track separated by $200 \mu \mathrm{m}$. A typical experiment would take $2-3 \mathrm{hr}$ to complete all nine tracks. DA neurons were identified using established electrophysiological criteria described by Grace and Bunney (1983). Specific electrophysiological characteristics include a slow irregular or bursting discharge pattern, an initial segment-somatodendritic break in the positive phase, and a long-duration (2-4 msec) biphasic action potential waveform. After an individual DA neuron was isolated, its spontaneous activity was recorded for 2-3 min. Three parameters of activity were sampled, the first being the number of spontaneously active DA neurons recorded per electrode track. The validity of this index as a reliable measure of DA neuron activity change has been discussed previously (West and Grace, 2000). This index has been shown to be reliable and consistent across animals and across laboratories (Bunney and Grace, 1978; Chiodo and Bunney, 1983; White and Wang, 1983). Furthermore, treatments that increase or decrease the number of spontaneously active DA neurons per electrode track have been shown to provoke corresponding changes in DA efflux (Moore et al., 1998). The other two parameters of activity that were sampled were the basal firing rate and the proportion of spikes fired by the DA neurons that occurred in bursts. The onset of a burst was defined as the occurrence of two spikes with an interspike interval $<80$ msec, and the termination of a burst was defined as the subsequent occurrence of an interspike interval $>160 \mathrm{msec}$ (Grace and Bunney, 1983). The percentage of spikes in bursts (\% SIB) was calculated by dividing the number of spikes occurring in bursts by the total number of spikes occurring in the same period of time.

Histology. At the end of each experiment, the recording site was marked via iontophoretic ejection of Pontamine sky blue dye from the tip of the recording electrode (30 $\mu \mathrm{A}$ constant current for 20-30 min). After dye ejection, brains were removed and fixed in formalin for at least $48 \mathrm{hr}$. The brains were then immersed in phosphate-buffered sucrose solution (25\%) until saturated. The tissue was sectioned into $40 \mu \mathrm{m}$ coronal slices, mounted, and stained with cresyl violet to enable histological determination of recording electrode and cannula infusion sites.

Data analysis. The number of spontaneously active DA neurons observed per electrode track, the basal firing rates of all DA neurons, and the $\%$ SIB were calculated as an average value for each rat and analyzed using three separate one-way ANOVAs with treatment group as the between-subjects factor. Multiple comparisons were made using twotailed Dunnett's tests. Analyses of the distribution of firing rates and \% SIB of all DA neurons across all rats in different treatment groups were assessed using Kolmogorov-Smirnov two-sample tests.

\section{RESULTS}

\section{Histology}

The locations of the tips of the cannulas were confirmed to lie within the vSub, the NAc, and the mPFC (Fig. 1). All rats had infusions that were localized primarily within the ventral CA1 and subicular regions of the ventral hippocampus, with some placements encroaching on the entorhinal cortex or medial dentate gyrus. The data from these rats did not differ from the data of rats with placements that were exclusively in the vSub. Infusions of kynurenic acid into the NAc were localized to the medial shell region, whereas infusions of TTX into the mPFC were localized to the prelimbic or infralimbic cortex. Both of these regions receive a dense projection from the vSub (Brog et al., 1993; Conde et al., 1995).

\section{Effects of infusions of NMDA or TTX into the vSub on DA neuron activity}

Inf usions of NMDA into the vSub caused a dramatic increase in the number of DA neurons encountered per electrode track. After inf usions of $0.25 \mu \mathrm{g}$ of NMDA, there was a $60.1 \%$ increase in the number of spontaneously active DA cells, and infusions of the $0.75 \mu \mathrm{g}$ dose caused a $111.2 \%$ increase relative to that of 


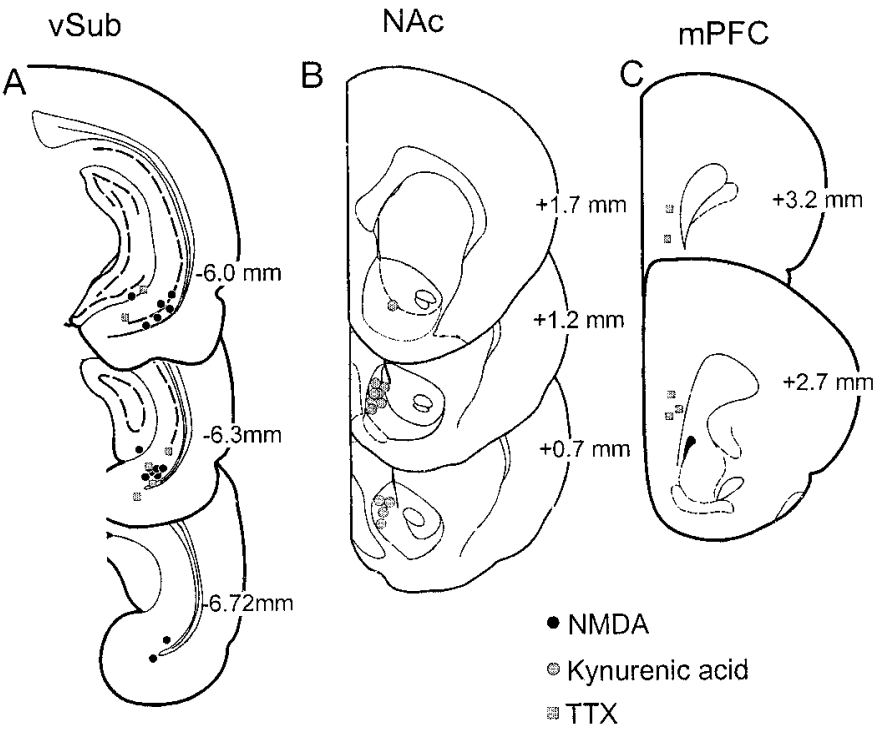

Figure 1. Histological verification of all recording and infusion sites in the present study. The location of infusions is shown for all rats receiving the following: $A$, infusions of $0.75 \mu \mathrm{g}$ of NMDA (black circles) or TTX (gray squares) into the vSub; $B$, inf usions of kynurenic acid into the NAc (gray circles); or $C$, infusions of TTX into the mPFC (gray squares). Plates are taken from Paxinos and Watson (1997), and the numbers beside each plate correspond to millimeters from bregma.

vehicle-treated animals. Overall, an infusion of NMDA into the vSub is the most potent stimulus for activating silent DA neurons reported to date.

Analysis of the number of spontaneously active DA neurons per electrode track for all treatment groups revealed a significant effect of treatment $\left(F_{(6,35)}=13.64 ; p<0.001\right.$; Figure $\left.2 A\right)$. Significant effects of treatment were also observed with respect to both the basal firing rate data $\left(F_{(6,35)}=4.12 ; p<0.005\right)$ and the $\% \operatorname{SIB}\left(F_{(6,35)}=3.926 ; p<0.005\right)$. Dunnett's tests revealed that infusions of either $0.25 \mu \mathrm{g}$ ( $n=6$ rats; 104 neurons) or $0.75 \mu \mathrm{g}$ ( $n=7$ rats; 162 neurons) of NMDA into the vSub increased the number of spontaneously active DA neurons per electrode track in a dose-dependent manner, relative to vehicle treatments $(n=$ 6 rats; 65 neurons) ( $p<0.05$ and 0.01 , respectively; Fig. $2 A)$. Interestingly, this occurred without a change in either the mean firing rate or the $\%$ SIB (Figs. $3 A, 4 A$, respectively). At least part of this effect may have been caused by activation of silent DA neurons that displayed activity at substantially lower firing rates, as suggested by differences in the firing rate distribution (Kolmogorov-Smirnov test, $p<0.01$; Fig. $3 C$ ). Thus, particularly with the low dose of NMDA, there was an apparent increase in the number of slowly firing DA neurons. The apparent lack of increase in slowly firing DA neurons after the $0.75 \mu \mathrm{g}$ dose of NMDA (compared with the $0.25 \mu \mathrm{g}$ dose) suggests that the higher dose of NMDA may have also increased the firing rates of DA neurons that would normally be spontaneously active at a lower firing rate after the lower dose of NMDA. A separate analysis revealed that there were no differences in the number of spontaneously active DA neurons encountered across all nine tracks $\left(F_{(8,24)}=0.87 ; \mathrm{NS}\right)$. Thus, stimulation of the vSub exerted a robust excitatory influence on the basal level of DA neuron firing in the VTA, as indicated by the increase in the number of spontaneously active DA neurons per electrode track that were observed after NMDA infusions into the vSub.

In contrast to the effects of NMDA, infusions of TTX into the
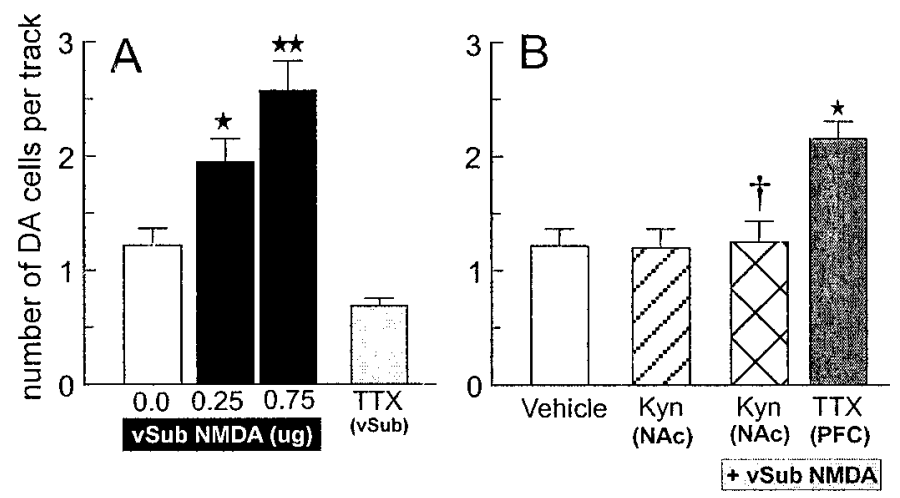

Figure 2. Infusions of NMDA into the vSub increase DA neuron population activity. $A$, Activation of the vSub led to a dose-dependent increase in spontaneously active DA neurons. The mean number of spontaneously active DA neurons (+SEM) recorded per electrode track from rats receiving vehicle infusions ( $n=6$ rats; white bar), 0.25 or 0.75 $\mu \mathrm{g}$ of NMDA ( $n=6$ rats, 7 rats, respectively; black bars), or TTX $(n=$ 6 rats; gray bar) into the vSub is shown. $B$, Blockade of glutamate transmission in the NAc prevented the activation of VTA DA neuron firing by vSub stimulation in a manner that was independent of the vSub-mPFC pathway. The mean number of spontaneously active DA neurons (+SEM) recorded per electrode track from rats receiving vehicle inf usions (same as $A$; white bar), infusions of kynurenic acid (Kyn) into the NAc in combination with vehicle infusions into the vSub $(n=6$ rats; hatched bar), infusions of kynurenic acid into the NAc in combination with $0.75 \mu \mathrm{g}$ of NMDA into the vSub ( $n=6$ rats; cross-hatched bar), or infusions of TTX into the mPFC in combination with $0.75 \mu \mathrm{g}$ of NMDA into the vSub ( $n=5$ rats; gray bar $)$ is shown. ${ }^{*} p<0.05$ and $* * p<0.01$ versus control; $\uparrow p<0.05$ versus the group receiving $0.75 \mu \mathrm{g}$ of NMDA.

$\operatorname{vSub}(n=6$ rats; 37 neurons) caused a trend toward a decrease in the number of spontaneously active DA cells per electrode track relative to vehicle treatments, but this difference was not statistically significant ( $p>0.10$; Fig. $2 A$ ). However, inf usions of TTX into the vSub caused a significant decrease in the basal firing rates of DA neurons $(p<0.05$; Fig. $3 A)$ and the $\%$ SIB $(p<0.05$; Fig. $4 A$ ). These data show that vSub also exerts a tonic excitatory influence over the firing characteristics of VTA DA neurons, as suggested by the decreases in both the firing rate and bursting activity of DA neurons observed after inactivation of the vSub by TTX.

\section{Involvement of the NAc and the MPFC in the regulation of DA neuron activity by the vSub}

Although there are no direct projections from the vSub to the VTA, there are several potential pathways via which the vSub could affect DA neuron firing. Thus, the vSub projects to both the mPFC and the NAc, which provide direct or indirect afferents to DA neurons (Brog et al., 1993; Conde et al., 1995). This was assessed by pharmacological manipulations of each of these systems. Infusions of kynurenic acid into the NAc in combination with vehicle infusions into the vSub ( $n=6$ rats; 64 neurons) did not alter significantly the number of spontaneously active DA neurons per electrode track (Fig. 2B). However, similar to the effects observed after TTX infusions into the vSub, administration of kynurenic acid into the NAc reduced both the firing rate $(p<0.01$; Fig. $3 B)$ and the $\%$ SIB $(p<0.01$; Fig. $4 B)$ of VTA DA neurons.

Infusions of the glutamate receptor antagonist kynurenic acid into the NAc completely abolished the increase in spontaneously active DA neurons per electrode track induced by infusions of $0.75 \mu \mathrm{g}$ of NMDA into the vSub ( $n=6$ rats; 68 neurons; 

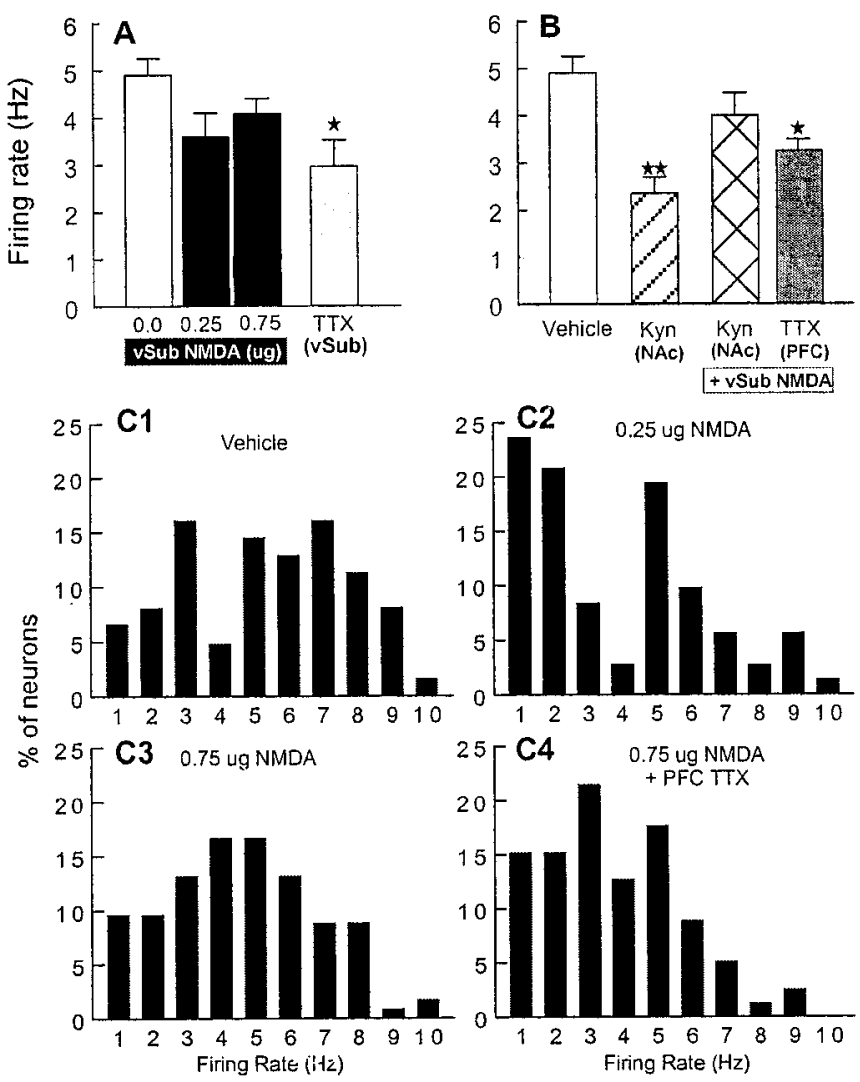

Figure 3. Modulation of DA neuron firing rate by the vSub, the NAc, and the mPFC. $A$, In contrast to the effects on spontaneous activity, activation of the vSub did not change significantly the VTA DA neuron firing rate. Nonetheless, inactivation of the vSub did decrease VTA DA cell firing. The mean firing rate of DA neurons (+SEM) recorded from rats receiving vehicle infusions (white bar), 0.25 or $0.75 \mu \mathrm{g}$ of NMDA (black bars), or TTX (gray bar) into the vSub is shown. $B$, Blockade of glutamate transmission in the NAc attenuates the DA neuron firing rate. The mean firing rate of DA neurons (+SEM) recorded from rats receiving vehicle inf usions (same as $A$; white bar), infusions of kynurenic acid into the NAc in combination with vehicle infusions into the vSub (hatched bar), infusions of kynurenic acid into the NAc in combination with $0.75 \mu \mathrm{g}$ of NMDA into the vSub (cross-hatched bar), or infusions of TTX into the mPFC in combination with $0.75 \mu \mathrm{g}$ of NMDA into the vSub (gray bar) is shown. ${ }^{*} p<0.05$ and ${ }^{*} p<0.01$ versus control. $C$, Distribution of mean firing rates of all DA neurons recorded from rats receiving vehicle injection $(C 1), 0.25 \mu \mathrm{g}$ of NMDA $(C 2)$ or $0.75 \mu \mathrm{g}$ of NMDA $(C 3)$ into the vSub, or infusions of TTX into the mPFC in combination with $0.75 \mu \mathrm{g}$ of NMDA into the vSub (C4) is shown. This suggests that the increase in DA cell spontaneous activity after vSub stimulation may be caused by the activation of slow-firing DA neurons.

Dunnett's, $p=1.0$; Fig. $2 B$ ). One planned comparison confirmed that inf usions of kynurenic acid into the NAc in combination with NMDA in the vSub resulted in significantly fewer spontaneously active DA neurons compared with NMDA treatments alone $[t(11)=4.04 ; p<0.005]$. This combination of treatments did not alter significantly either the firing rate (Fig. $3 B$ ) or the $\% \mathrm{SIB}$ (Fig. $4 B$ ) of the DA neurons sampled.

In contrast, inactivation of the mPFC by infusions of TTX, in combination with stimulation of the vSub with $0.75 \mu \mathrm{g}$ of NMDA ( $n=5$ rats; 75 neurons), did not block the increase in the number of spontaneously active DA neurons produced by NMDA infusions into the vSub $(p<0.05$; Fig. $2 B)$. One planned comparison confirmed that the number of spontaneously active DA neurons observed in the mPFC TTX and vSub NMDA group did not
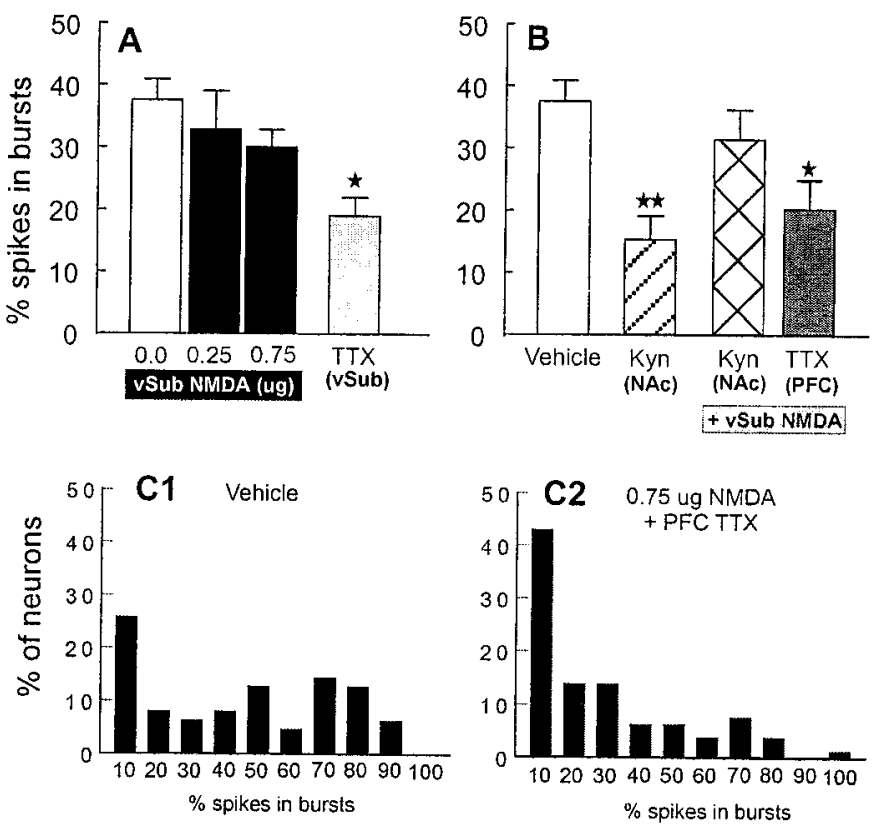

Figure 4. Modulation of DA neuron burst firing by the vSub, the NAc, and the mPFC. $A$, As with firing rate, activation of the vSub did not cause a significant change in DA cell burst firing, although inactivation of the vSub did attenuate bursting. The mean \% SIB of DA neurons (+SEM) recorded from rats receiving vehicle infusions (white bar), 0.25 or $0.75 \mu \mathrm{g}$ of NMDA (black bars), or TTX (gray bar) into the vSub is shown. $B$, Similarly, glutamate transmission in the NAc also controls basal levels of burst firing of VTA DA neurons. The mean \% SIB of DA neurons (+SEM) recorded from rats receiving vehicle infusions (same as $A$; white bar), infusions of kynurenic acid into the NAc in combination with vehicle infusions into the vSub (hatched bar), infusions of kynurenic acid into the NAc in combination with $0.75 \mu \mathrm{g}$ of NMDA into the vSub (cross-hatched bar), or inf usions of TTX into the MPFC in combination with $0.75 \mu \mathrm{g}$ of NMDA into the vSub (gray bar) is shown. ${ }^{*} p<0.05$ and ${ }^{*} p<0.01$, versus control. $C$, Distribution of $\%$ SIB of all DA neurons recorded from rats receiving vehicle injection $(\mathrm{Cl})$ or inf usions of TTX into the mPFC in combination with $0.75 \mu \mathrm{g}$ of NMDA into the vSub $(C 2)$ is shown.

differ from that with vSub NMDA treatment alone $[t(10)=1.33$; $p>0.20, \mathrm{NS}]$. However, this combination of treatments did reduce significantly the basal firing rate and the \% SIB recorded from DA neurons $(p<0.05)$. Accordingly, inactivation of the $\mathrm{mPFC}$ by TTX resulted in a significant difference in the distributions of both the firing rate and \% SIB of DA neurons (Kolmogorov-Smirnov test, $p<0.01$ ), increasing the proportion of neurons that displayed firing rates of $<3 \mathrm{~Hz}$ (Fig. 3C1,C4) and the proportion of neurons that displayed $<10 \%$ of their spikes in bursts (Fig. 4C1,C2). This supports a role for the mPFC in the regulation of basal firing characteristics of spontaneously active DA neurons and confirms that infusions of TTX into the mPFC were sufficient to reduce activity in this cortical region, as indicated by the decrease in firing rates and burst firing of these neurons. Thus, the increase in DA neuron activity, induced by NMDA stimulation of the vSub, is mediated primarily by glutamate receptor activity in the NAc, but not by neural activity of the mPFC. Moreover, like neural activity in the vSub, glutamate receptor tone in the NAc exerts a tonic excitatory influence over the firing characteristics of VTA DA neurons, because blockade of these receptors decreases both the firing rate and bursting activity of DA neurons, without influencing the number of spontaneously active DA neurons. 


\section{DISCUSSION}

The present study demonstrates that the vSub exerts a powerful control over the activity of DA neurons in the VTA and does so via glutamatergic mechanisms localized within the NAc. NMDAinduced stimulation of the vSub dramatically increased the number of spontaneously active DA neurons per electrode track, which was completely abolished after glutamate receptor blockade in the NAc. This effect was not mediated by the hippocampal-cortical-accumbens pathway, because inf usions of TTX into the mPFC had no effect on the NMDA-induced increase in DA neuron population activity. In addition, the vSub also exerts a tonic influence (i.e., the basal steady-state level of activity that is present in the absence of vSub activation) over the firing rate and burst firing of VTA DA neurons. Inactivation of the vSub or glutamate receptor blockade in the NAc decreased both of these parameters. In view of these data, it is likely that the changes in mesoaccumbens DA release after chemical stimulation of the vSub (Brudzynski and Gibson, 1997; Legault and Wise, 1999; Legault et al., 2000) may have been mediated, in part, by increased firing of VTA DA neurons. It is interesting to note that basal striatal DA efflux has been shown previously to correlate strongly with DA neuron population activity but not to correlate with either the average firing rate or burst firing level of individual nigrostriatal DA neurons of drug-naïve rats (Moore et al., 1998).

\section{Neural pathways mediating vSub control of VTA DA neuron population activity}

There are several projection pathways that can potentially mediate subicular regulation of VTA DA cell firing. The observation that infusions of the glutamate receptor antagonist kynurenic acid into the NAc completely abolished the increase in VTA DA neuron population activity suggests that this pathway involves glutamatergic afferents to GABAergic medium spiny projection neurons of the NAc. Moreover, the lack of effect of mPFC inactivation on this response suggests that these effects are mediated by a direct vSub-NAc projection (Groenewegen et al., 1987). Anatomical and electrophysiological studies point to at least two potential efferent pathways by which the firing activity of NAc projection neurons could modulate DA cell body activity, the first being a direct projection from the NAc to the VTA (Zahm and Heimer, 1990; Kalivas et al., 1993). However, it is unlikely that this projection mediates the increases in DA neuron activity, because this projection is primarily GABAergic (Kalivas et al., 1993) and electrophysiological studies have shown that stimulation of the NAc evokes short-latency inhibitory responses in VTA neurons (Maeda and Mogenson, 1980).

A more likely route by which activity in the hippocampalventral striatal pathway may modulate DA neuron firing is via the ventral pallidum (VP). The NAc sends a dense GABA projection to the VP, and stimulation of either the NAc or its glutamatergic afferents (including the vSub) can inhibit VP neuronal firing (Jones and Mogenson, 1980; Tsai et al., 1985; Yang and Mogenson, 1985, 1987; Chrobak and Napier, 1993). In contrast to the medium spiny neurons of the NAc, the GABAergic output neurons of the VP are characterized by relatively high rates of spontaneous activity (Jones and Mogenson, 1980; Yang and Mogenson, 1985, 1987; Mogenson et al., 1993), thereby exerting a tonic inhibitory influence over efferent structures. The VP can influence DA neural activity via a direct projection to the VTA (Swanson et al., 1984; Tsai et al., 1985; Zahm and Heimer, 1990; Mogenson et al., 1993). Although the precise mechanisms by

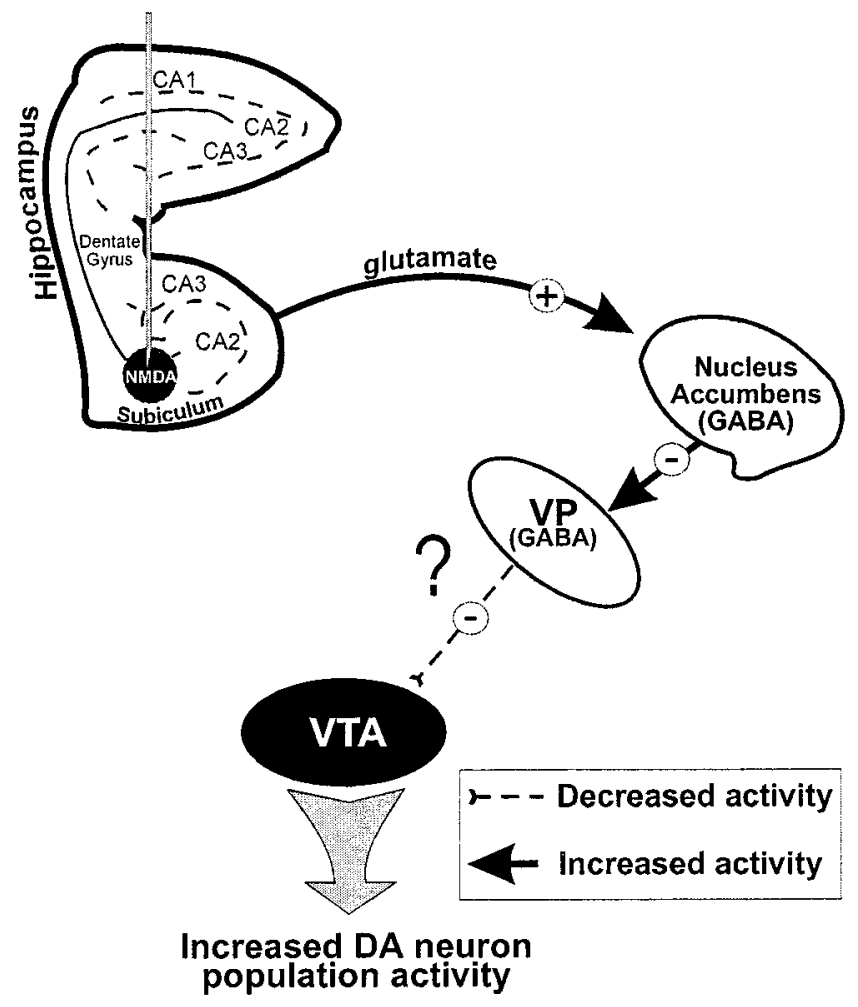

Figure 5. A model describing the neural circuitries by which the vSub may modulate DA neuron activity. Stimulation of the vSub by inf usions of NMDA activates glutamatergic afferents to the NAc. This in turn is proposed to inhibit the activity of neurons in the VP. As a result, there is a removal of tonic inhibition of neurons in the VTA. In this model, we propose that the VP-VTA projection provides sufficient inhibitory influence to hold a subpopulation of DA neurons in an inactive state. Thus, inhibition of VP firing would lead to an increase in the number of VTA DA neurons firing spontaneously. The question mark beside the VP to VTA path indicates that, although this pathway is known to be present in the rat, its involvement in this response remains speculative at the present time.

which this could alter VTA DA cell activity in the manner observed here are not known at present, one potential mechanism that may account for these findings is that stimulation of the vSub would have increased firing of GABAergic projection neurons of the NAc, causing a decrease in VP neural activity. The decrease in VP activity would then be expected to cause a reduction of the GABAergic inhibition over the VTA. It is known that the spontaneous activity of DA neurons is not dependent on glutamatergic afferents but instead is driven by an endogenous pacemaker conductance (Grace and Bunney, 1983; Grace and Onn, 1989; Kitai et al., 1999). Therefore, we propose that the increase in DA neuron population activity may be caused by a release from VP GABAergic inhibition (Fig. 5). This activation of normally silent DA neurons may contribute to the increase in slow firing, spontaneously active DA cells observed after NMDA inf usions into the vSub. We are currently performing experiments to evaluate this hypothesis.

The present data suggest that the glutamatergic vSub-NAc pathway also plays a role in regulating the firing parameters of DA neurons. Inactivation of the vSub with TTX or infusions of kynurenic acid into the NAc decreased both the firing rate and \% SIB of DA neurons without decreasing the number of spontaneously active DA neurons. These effects are similar to those 
observed after transection of striatal outputs to nigrostriatal DA neurons, which also caused a decrease in firing parameters of A9 DA neurons (Pucak and Grace, 1994). Although the mechanism by which the basal activity in the vSub projection to the NAc may regulate firing characteristics of DA neurons remains unclear at the present time, one possibility is that it may involve outputs from the NAc to the VP and subsequently to the pedunculopontine tegmental nucleus (PPTg). The VP sends projections to the PPTg (Swanson et al., 1984; Tsai et al., 1985; Zahm and Heimer, 1990; Mogenson et al., 1993), and previous studies have shown that the PPTg exerts excitatory control over both the firing activity of DA neurons (Kelland et al., 1993; Lokwan et al., 1999) and mesoaccumbens DA efflux via glutamatergic and cholinergic mechanisms in the VTA (Blaha et al., 1996; Forster and Blaha, 2000). Reducing the activity of NAc neurons, either by TTX in the vSub or kynurenic acid in the NAc, would cause an increase in VP firing. The finding that these manipulations also attenuate burst firing, which has been shown to be dependent on glutamatergic afferents in vivo (Overton and Clark, 1997; Kitai et al., 1999), suggests that these alterations may be caused by increased inhibition of PPTg neurons, which would decrease VTA burst firing.

These data provide further support for the hypothesis that distinct afferent pathways regulate DA cell population activity and firing rate and burst firing of DA neurons (Pucak and Grace, 1994). This dual regulation of DA neuron activity may be mediated by an interplay between glutamate and GABA afferents projecting to DA neurons. Thus, in those DA neurons that are inactive because of a hyperpolarization by VP afferents, one would expect little effect of glutamate, because (1) glutamate acts primarily on NMDA receptors on mesolimbic DA neurons (Karreman et al., 1996) and (2) DA neurons hyperpolarized by VP GABAergic afferents would be unresponsive to NMDA receptor stimulation caused by $\mathrm{Mg}^{2+}$ blockade (Mayer et al., 1984). Therefore, glutamate afferents would only be capable of increasing the firing rate and burst firing of DA neurons that were previously spontaneously active. However, after the DA neurons became spontaneously active via removal of the VP GABAergic inhibition (as would be achieved by activation of the vSub-NAc pathway), the glutamatergic afferents could then augment their firing rate and burst firing.

The fact that inf usions of TTX into the mPFC did not block the increase in DA neuron population activity induced by infusions of NMDA into the vSub was not surprising, because the proportion of A10 DA neurons that receive mPFC input represents a minority of the total neuronal population (Swanson, 1982; Carr and Sesack, 2000). Moreover, DA neurons that receive mPFC input do not project to the NAc but instead send reciprocal projections back to the mPFC (Carr and Sesack, 2000). The observed reduction in burst firing and the firing rate of VTA DA neurons after infusions of TTX into the mPFC is consistent with a number of studies showing that cooling or infusions of local anesthetics into the mPFC decrease burst firing of DA neurons (Svensson and Tung, 1989; Murase et al., 1993), whereas electrical or chemical stimulation of this region increases bursting activity by glutamate-dependent mechanisms (Murase et al., 1993; Tong et al., 1996). Taken together, the present findings, in addition to previous data, provide important insight regarding dissociable mechanisms of hippocampal and cortical regulation of DA neuron activity.

\section{Modulation of mesoaccumbens DA release by the vSub}

The present findings complement previous studies showing that stimulation of the vSub can increase mesoaccumbens DA efflux. Infusions of NMDA into the vSub, similar to those used in the present study, produced a substantial increase in NAc DA extracellular levels (Legault and Wise, 1999; Legault et al., 2000), an effect that was abolished by reverse dialysis of glutamate receptor antagonists into the VTA. This observation complements the present data, in which stimulation of hippocampal afferents to the NAc can increase the population activity of DA neurons, which presumably can enhance mesoaccumbens DA efflux. However, this does not exclude the potential role of glutamate-mediated DA release occurring directly in the ventral striatum. Thus, blockade of NMDA receptors in the NAc will block the increase in DA efflux evoked by electrical stimulation of the vSub (Blaha et al., 1997; Taepavarapruk et al., 2000), although NMDA receptors play only a minor role in the glutamate-evoked activity of NAc neurons (Pennartz et al., 1991; Hu and White, 1996). Furthermore, it is known that NMDA receptors are located on intervaricose segments of tyrosine hydroxylase-containing neuronal processes in the NAc shell (Gracy and Pickel, 1996). Therefore, it is likely that the vSub modulation of DA release in the NAc may be the result of both a presynaptic mechanism localized within the NAc and a polysynaptic mechanism involving the NAc, VP, and VTA. The vSub would thus be in a unique position to regulate both the tonic and phasic release of DA, the former by presynaptic modulation of DA terminal release (Grace, 1991; Blaha et al., 1997; Taepavarapruk et al., 2000) and the latter by a polysynaptic circuit linking the NAc to the VTA (Zahm and Heimer, 1990; Grace, 1991).

\section{Implications for schizophrenia}

Dysfunction of the DA system has long been implicated as a primary factor in the pathophysiology of schizophrenia. More recent studies have suggested that there may be a developmental pathology of the hippocampus in this disorder (Lipska and Weinberger, 2000), particularly in the ventral regions that project to limbic structures. The present study shows that the vSub is involved in regulating the basal firing characteristics as well as the overall activity level of VTA DA neurons in the normal rat. If this system is disrupted, particularly early in development, it is likely that tonic control of VTA DA neuron activity would be affected. Under such conditions, stimuli that cause phasic activation of DA neuron firing may be expected to have an abnormally large impact on impulse-dependent DA release in limbic structures (Grace, 1991). This could contribute to the heightened DA responsivity observed in rats with developmental disruptions of ventral hippocampal circuits (Grace, 2000; Lipska and Weinberger, 2000).

\section{REFERENCES}

Bardgett ME, Henry JD (1999) Locomotor activity and accumbens fos expression driven by ventral hippocampal stimulation require D1 and D2 receptors. Neuroscience 94:59-70.

Blaha CD, Allen LF, Das S, Ingles WL, Latimet MP, Vincent SR, Winn P (1996) Modulation of dopamine efflux in the nucleus accumbens after cholinergic stimulation of the ventral tegmental area in intact, pedunculopontine tegmental nucleus-lesioned and laterodorsal tegmental nucleus-lesioned rats. J Neurosci 16:714-722.

Blaha CD, Yang CR, Floresco SB, Barr AM, Phillips AG (1997) Stimulation of the ventral subiculum of the hippocampus evokes glutamate receptor-mediated changes in dopamine efflux in the rat nucleus accumbens. Eur J Neurosci 5:902-911. 
Bogerts B (1993) Recent advances in the neuropathology of schizophrenia. Schizophr Bull 19:431-445.

Brog JS, Salyapongse A, Deutch AY, Zahm DS (1993) The patterns of afferent innervation of the core and shell in the "accumbens" part of the rat ventral striatum: immunohistochemical detection of retrogradely transported fluoro-gold. J Comp Neurol 338:255-278.

Brudzynski SM, Gibson CJ (1997) Release of dopamine in the nucleus accumbens caused by stimulation of the subiculum in freely moving rats. Brain Res Bull 42:303-308.

Bunney BS, Grace AA (1978) Acute and chronic haloperidol treatment: comparison of effects on nigral dopaminergic cell activity. Life Sci 23:1715-1728.

Burns LH, Robbins TW, Everitt BJ (1993) Differential effects of excitotoxic lesions of the basolateral amygdala, ventral subiculum and medial prefrontal cortex on responding with conditioned reinforcement and locomotor activity potentiated by intra-accumbens infusions of D-amphetamine. Behav Brain Res 55:167-183.

Burns LH, Annett L, Kelley AE, Everitt BJ, Robbins TW (1996) Effects of lesions to amygdala, ventral subiculum, medial prefrontal cortex, and nucleus accumbens on the reaction to novelty: implication for limbicstriatal interactions. Behav Neurosci 110:60-73.

Carr DB, Sesack SR (2000) Projections from the rat prefrontal cortex to the ventral tegmental area: target specificity in the synaptic associations with mesoaccumbens and mesocortical neurons. J Neurosci 20:3864-3873.

Chiodo LA, Bunney BS (1983) Typical and atypical neuroleptics: differential effects of chronic administration on the activity of A9 and A10 midbrain dopaminergic neurons. J Neurosci 3:1607-1619.

Chrobak JJ, Napier TC (1993) Opioid and GABA modulation of accumbens-evoked ventral pallidal activity. J Neural Transm Gen Sect 93:123-143.

Conde F, Maire-Lepoivre E, Audinat E, Crepel F (1995) Afferent connections of the medial frontal cortex of the rat. II. Cortical and subcortical afferents. J Comp Neurol 325:567-593.

DeFrance JF, Sikes RW, Chronister RB (1985) Dopamine action in the nucleus accumbens. J Neurophysiol 54:1568-1577.

Floresco SB, Phillips AG (1999) Dopamine and hippocampal input to the nucleus accumbens play an essential role in the search for food in an unpredictable environment. Psychobiol 27:227-286.

Floresco SB, Seamans JK, Phillips AG (1996) A selective role for dopamine in the nucleus accumbens of the rat in random foraging but not delayed spatial win-shift foraging. Behav Brain Res 80:161-168.

Forster GL, Blaha CD (2000) Laterodorsal tegmental stimulation elicits dopamine efflux in the rat nucleus accumbens by activation of acetylcholine and glutamate receptors in the ventral tegmental area. Eur J Neurosci 12:3596-3604.

Gonon F, Sundstrom L (1996) Excitatory effects of dopamine released by impulse flow in the rat nucleus accumbens in vivo. Neuroscience 75:13-18.

Grace AA (1991) Phasic versus tonic dopamine release and the modulation of dopamine system responsivity: a hypothesis for the etiology of schizophrenia. Neuroscience 41:1-24.

Grace AA (2000) Gating of information flow in the limbic system and the pathophysiology of schizophrenia. Brain Res Rev 31:330-341.

Grace AA, Bunney BS (1983) Intracellular and extracellular electrophysiology of nigral dopaminergic neurons. 1. Identification and characterization. Neuroscience 10:301-315.

Grace AA, Onn S-P (1989) Morphological and electrophysiological properties of immunohistochemically identified rat dopamine neurons recorded in vitro. J Neurosci 9:3463-3481.

Gracy KN, Pickel VM (1996) Ultrastructural immunocytochemical localization of the $N$-methyl-D-aspartate receptor and tyrosine hydroxylase in the shell of the rat nucleus accumbens. Brain Res 739:169-181.

Groenewegen HJ, Vermeulen-Van der Zee E, Te Kortschot A, Witter MP (1987) Organization of the projections from the subiculum to the ventral striatum in the rat. A study using anterograde transport of Phaseolus vulgarus leucoagglutinin. Neuroscience 23:103-120.

Groenewegen HJ, Berendse HW, Meredith GE, Haber SN, Voorn P, Wolters JG, Lohman AHM (1991) Functional anatomy of the ventral, limbic system innervated striatum. In: The mesolimbic dopamine system (Willner P, Scheel-Kruger J, eds), pp 19-59. New York: Wiley.

Hu XT, White FJ (1996) Glutamate receptor regulation of rat nucleus accumbens neurons in vivo. Synapse 23:208-218.

Jones DL, Mogenson GJ (1980) Nucleus accumbens to globus pallidus GABA projection: electrophysiological and iontophoretic investigations. Brain Res 188:93-105.

Kalivas PW, Churchill L, Klitenick MA (1993) GABA and enkephalin projection from the nucleus accumbens and ventral pallidum to the ventral tegmental area. Neuroscience 57:1047-1060

Karreman M, Westerink BH, Moghaddam B (1996) Excitatory amino acid receptors in the ventral tegmental area regulate dopamine release in the ventral striatum. J Neurochem 67:601-607.

Kelland MD, Freeman AS, Rubin J, Chiodo LA (1993) Ascending afferent regulation of rat midbrain dopamine neurons. Brain Res Bull $31: 539-546$.
Kitai ST, Shepard PD, Callaway JC, Scrogges R (1999) Afferent modulation of dopamine neuron firing patterns. Curr Opin Neurobiol 9:690-697.

Legault M, Wise RA (1999) Injections of $N$-methyl-D-aspartate into the ventral hippocampus increase extracellular dopamine in the ventral tegmental area and nucleus accumbens. Synapse 31:241-249.

Legault M, Rompré PP, Wise RA (2000) Chemical stimulation of the ventral hippocampus elevates nucleus accumbens dopamine by activating dopaminergic neurons of the ventral tegmental area. J Neurosci 20:1635-1642.

Lipska BK, Weinberger DR (2000) To model a psychiatric disorder in animals: schizophrenia as a reality test. Neuropsychopharmacology 23:223-239.

Lokwan SJA, Overton PG, Berry MS, Clark D (1999) Stimulation of the pedunculopontine tegmental nucleus in the rat produces burst firing in A9 dopaminergic neurons. Neuroscience 92:245-254.

Maeda H, Mogenson GJ (1980) An electrophysiological study of inputs to neurons of the ventral tegmental area from the nucleus accumbens and medial preoptic-anterior hypothalamic areas. Brain Res 197:365-377.

Mayer ML, Westbrook GL, Guthrie PB (1984) Voltage-dependent block by $\mathrm{Mg}^{2+}$ of NMDA responses in spinal cord neurones. Nature 309:261-263.

Mogenson GJ, Brudzynski SM, Wu M, Yang CY, Yim CCY (1993) From motivation to action: a review of dopaminergic regulation of limbic to nucleus accumbens to ventral pallidum to pedunculopontine nucleus circuitries involved in limbic-motor integration. In: Limbic motor circuits in neuropsychiatry (Kalivas PW, Barnes CD, eds), pp 193-236. Boca Raton, FL: CRC.

Moore H, Todd CL, Grace AA (1998) Striatal extracellular dopamine levels in rats with haloperidol-induced depolarization block of substantia nigra dopamine neurons. J Neurosci 18:5068-5077.

Murase S, Grenhoff J, Chouvet G, Gonon FG, Svensson TH (1993) Prefrontal cortex regulates burst firing and transmitter release in rat mesolimbic dopamine neurons studied in vivo. Neurosci Lett 157:53-56.

Overton PG, Clark D (1997) Burst firing in midbrain dopaminergic neurons. Brain Res Rev 25:312-334.

Paxinos G, Watson C (1997) The rat brain in stereotaxic coordinates, Ed 3. San Diego: Academic.

Pennartz CM, Boeijinga PH, Kitai ST, Lopes da Silva FH (1991) Contribution of NMDA receptors to postsynaptic potentials and pair-pulse facilitation in identified neurons of the rat nucleus accumbens in vitro. Exp Brain Res 86:190-198.

Pennartz CMA, Dolleman-vender Weel MJ, Kitai ST, Lopes Da Silva FH (1992) Presynaptic dopamine $D_{1}$ receptors attenuate excitatory and inhibitory inputs to the shell region of the rat nucleus accumbens studied in vitro. J Neurophysiol 67:1325-1333.

Pennartz CMA, Groenewegen HJ, Lopes Da Silva FH (1994) The nucleus accumbens as a complex of functionally distinct neuronal ensembles: an integration of behavioural, electrophysiological and anatomical data. Prog Neurobiol 42:719-761.

Pucak ML, Grace AA (1994) Evidence that systemically administered dopamine antagonists activate dopamine neuron firing primarily by blockade of somatodendritic autoreceptors. J Pharmacol Exp Ther 271:1181-1192.

Sesack SR, Pickel VM (1990) In the rat medial nucleus accumbens, hippocampal and catecholaminergic terminals converge on spiny neurons and are in apposition to each other. Brain Res 527:266-279.

Svensson T, Tung C-S (1989) Local cooling of pre-frontal cortex induces pacemaker-like firing of dopamine neurons in rat ventral tegmental area in vivo. Acta Physiol Scand 136:135-136.

Swanson LW (1982) The projections of the ventral tegmental area and adjacent regions: a combined fluorescent retrograde tracer and immunofluorescence study in the rat. Brain Res Bull 9:321-353.

Swanson LW, Mogenson GJ, Gerfen CR, Robinson P (1984) Evidence for a projection from the lateral preoptic area and substantia innominata to the "mesencephalic locomotor region" in the rat. Brain Res 295:161-178.

Taepavarapruk P, Floresco SB, Phillips AG (2000) Hyperlocomotion and increased dopamine efflux in the nucleus accumbens evoked by electrical stimulation of the ventral subiculum: role of ionotropic glutamate and dopamine $\mathrm{D}_{1}$ receptors. Psychopharmacology (Berl) $151: 242-251$

Tong ZY, Overton PG, Clark D (1996) Antagonism of NMDA receptors but not AMPA/kainate receptors blocks bursting in dopaminergic neurons induced by electrical stimulation of the prefrontal cortex. J Neural Transm 103:889-904.

Totterdell S, Smith AD (1989) Convergence of hippocampal and dopaminergic input onto identified neurons in the nucleus accumbens of the rat. J Chem Neuroanat 2:285-298.

Tsai CT, Mogenson GJ, Wu M, Yang CR (1985) A comparison of the effects of electrical stimulation of the amygdala and hippocampus on subpallidal output neurons to the pedunculopontine nucleus. Brain Res 494:22-29. 
West AR, Grace AA (2000) Striatal nitric oxide signaling regulates the neuronal activity of midbrain dopamine neurons. J Neurophysiol 83:1796-1808.

White FJ, Wang RY (1983) Differential effects of classical and atypical antipsychotic drugs on A0 and A10 dopamine neurons. Science 221:1054-1057.

Wu M, Brudzynski SM (1995) Mesolimbic dopamine terminals and locomotor activity induced from the ventral subiculum. NeuroReport 6:1601-1604.

Yang CR, Mogenson GJ (1984) Electrophysiological responses of neurones in the nucleus accumbens to hippocampal stimulation and the attenuation of the excitatory responses by the mesolimbic dopaminergic system. Brain Res 324:69-84.
Yang CR, Mogenson GJ (1985) An electrophysiological study of the neural projection from the hippocampus to the ventral pallidum and subpallidal areas by way of the nucleus accumbens. Neuroscience 15:1015-1024.

Yang CR, Mogenson GJ (1986) Dopamine enhances terminal excitability of hippocampal-accumbens neurons via D2 receptor: role of dopamine in presynaptic inhibition. J Neurosci 6:2470-2478.

Yang CR, Mogenson GJ (1987) Hippocampal signal transmission to the pedunculopontine nucleus and its regulation by dopamine D2 receptors in the nucleus accumbens: an electrophysiological and behavioural study. Neuroscience 23:1041-1055.

Zahm DA, Heimer L (1990) Two transpallidal pathways originating in the nucleus accumbens. J Comp Neurol 302:437-446. 